\title{
RESPONSE OF BLADED DISKS WITH MISTUNED BLADE-DISK INTERFACES
}

\author{
Javier Avalos \\ Arizona State University \\ Dept of Mechanical and \\ Aerospace Engineering \\ Tempe, Arizona 85287-6106, \\ USA \\ Tel.: (480) 965-4294 \\ Fax: (480) 965-1384 \\ javier.avalos@asu.edu
}

\author{
Marc P. Mignolet \\ Arizona State University \\ Dept of Mechanical and \\ Aerospace Engineering Tempe, \\ Arizona 85287-6106, USA \\ Tel.: (480) 965-1484 \\ Fax: (480) 965-1384 \\ marc.mignolet@asu.edu
}

\author{
Christian Soize \\ Université Paris-Est, \\ Laboratoire Modelisation et \\ Simulation Multi Echelle, \\ 5, Bd Descartes, 77454 \\ Marne-la-Vallée Cedex 02, \\ France \\ christian.soize@univ-paris-est.fr
}

\begin{abstract}
This paper focuses on the determination of the effects on the bladed disk forced response of small variations (mistuning) in the blade-disk interface properties as may result from blade seating. A blade-interfacedisk mean model is first developed that relies on both the Craig-Bampton approach and a local modeling of the interface. Then, both model and data uncertainties are introduced in this model using the nonparametric stochastic modeling approach. An example of application is presented that indicates a difference in physical behavior of a bladed disk with mistuned interfaces as compared to one with mistuned blade alone frequencies. Most notably, it is shown that at equal variability on the blade frequencies, the variations in blade-disk interface properties lead to a higher amplification factor.

Keywords: mistuning, interface variations, bladed disk, nonparametric
\end{abstract} stochastic modeling, random matrices, blade vibration

\section{INTRODUCTION}

The effects of blade-to-blade variations in their structural and geometrical properties, collectively referred to as mistuning, on the free and forced responses of bladed disks have been the subject of a large number of investigations in the last four decades. These efforts have led in particular to a good understanding (e.g. see [1-3]) of the physics underlying the localization of the free response and the amplification of the forced response. Computationally efficient reduced order modeling strategies (e.g. see [4-7]) have also been formulated and validated. Further, the largest possible amplification of the response and the conditions under which it occurs have been well analyzed (e.g. see [815]). More recently, multi-stage effects have also started being analyzed (e.g., see [16-19])

In all of the above investigations, mistuning has been assumed to originate from differences in the blades, be it in their geometrical and/or material properties. More rarely is the seating of an inserted blade in its fir tree/dove tail also mentioned. Note however that this variability does not affect the blade alone as other mistuning sources but it also affects the disk dynamics as the blade-disk interface is shared.

In this light, the focus of the present investigation is on the development of a formulation for the consideration of variability in the disk-blade interface and on the study of its effects on the entire disk response. Most notably, the present effort will assess whether the variability in interface properties exhibits similar or different properties than blade mistuning.

\section{BLADE-INTERFACE-DISK MODELING}

A detailed, finite element analysis of the effects of blade-disk interface variability, e.g. from blade seating, would be a very complex and computationally intensive effort as one would in particular have to vary the meshing at the blade root to model the seating variability. Accordingly, it would be very desirable to adopt a more global approach, i.e. a modeling of the blade-interface-disk system in which the interface is a clearly delineated component. Further, because Monte Carlo simulations will be undertaken to obtain a statistical perspective of the effects of interface variability, it will be important to dispose of a computationally efficient model.

The Craig-Bampton method of component mode synthesis is a particularly attractive approach for such an effort as it expresses the response of a structure in terms of the motion of its boundary (i.e., the blade-disk interface) and the modes of the structure with fixed interface, e.g., the clamped modes of the blade (see for example [5] for a prior application in bladed disks). This method will be applied here to both the blades and the disk separately and then the two models will be interfaced.

Consider first a typical blade (blade $b$ ) and partition its degrees-offreedom in terms of internal $(I)$ and boundary $(B)$ ones yielding the physical stiffness and mass matrices 


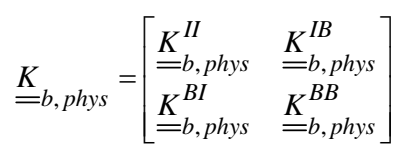

and

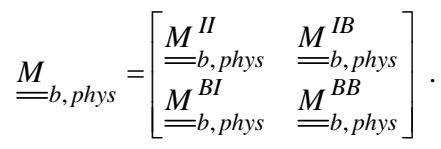

Following the Craig-Bampton approach, the internal $\left(\underline{X}_{b, p h y s}^{I}\right)$ and boundary ( $\underline{X}_{b, \text { phys }}^{B}$ ) degrees-of-freedom will be expressed as

$$
\underline{X}_{b, p h y s}^{I}=\underline{\Phi} b \underline{q}_{b}+\underline{\Xi}_{b} \underline{Y}_{b}
$$

and

$$
\underline{X}_{b, p h y s}^{B}=\underline{Y}_{b}
$$

where $\stackrel{\Phi}{=}$ denotes the modal matrix of $p$ selected modes of the clamped blade $b$, i.e. $\underline{\Phi}_{b}=\left[\underline{\phi}_{b, 1} \underline{\phi}_{b, 2} \cdots \underline{\phi}_{b, p}\right]$, satisfying

$$
\underline{K}_{b, \text { phys }}^{I I} \underline{\phi}_{b, j}=\omega_{b, j}^{2} \underline{M}_{b, \text { phys }}^{I I} \underline{\phi}_{b, j} \text {. }
$$

Further, in Eq. (3), the symbol $\Xi_{b}$ denotes the matrix of constraint modes

$$
\stackrel{\Xi}{=}_{b}=-\left(\underline{K}_{b, p h y s}^{I I}\right)^{-1} \underline{K}_{b, \text { phys }}^{I B} .
$$

Finally, the vector $\underline{q}_{b}$ denotes the generalized coordinates of the modes of the clamped blade $b$.

To the change of variables, from $\left(\underline{X}_{b, \text { phys }}^{I}, \underline{X}_{b, \text { phys }}^{B}\right)$ to $\left(\underline{q}_{b}, \underline{Y}_{b}\right)$, is accompanied by the transformation matrix

$$
\stackrel{T}{=} b_{b}=\left[\begin{array}{cc}
\stackrel{\Phi}{=} b & \stackrel{\Xi}{=} b \\
\underline{0} & \underline{I}
\end{array}\right]
$$

and thus, the corresponding stiffness and mass matrices of the blades are

$$
\stackrel{K=}{=} b, C B=T^{T}{ }_{b}^{K}=b, \text { phys } \stackrel{T}{=} b=\left[\begin{array}{cc}
K_{b, C B}^{q q} & 0 \\
0 & K^{Y Y} b, C B
\end{array}\right]
$$

and

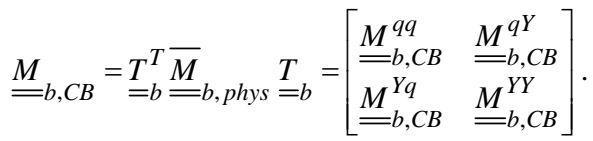

Since the reduced order model is built on the modal matrix $\stackrel{\Phi}{=}_{b}$, the matrices $\underset{=b, C B}{K^{q q}}$ and $\stackrel{M^{q q, C B}}{q q}$ are diagonal, and more specifically with nonzero elements equal to the natural frequencies and 1 if the modes $\underline{\phi}_{b, j}$ have been normalized with respect to the mass matrix $\underline{M}_{b \text {,phys }}^{I I}$.

A similar procedure can be followed with the disk to describe its response in terms of the motion of its degrees-of-freedom at its interface with every one of the $N$ blades, i.e. $\underline{Y}_{d}^{T}=\left[\underline{Y}_{d, 1}^{T} \underline{Y}_{d, 2}^{T} \cdots \underline{Y}_{d, N}^{T}\right]$, and of the modal coordinates $\underline{q}_{d}$ associated with the disk modes with fixed blade interface $\underline{\phi}_{d, l}$ stacked in the modal matrix $\underset{\Phi_{d}}{=}=\left[\underline{\phi}_{d, 1} \underline{\phi}_{d, 2} \cdots \underline{\phi}_{d, p}\right]$. The disk stiffness and mass matrices in the coordinates $\left(\underline{q}_{d}, \underline{Y}_{d}\right)$ are then

$$
\underline{K}_{d, C B}=\left[\begin{array}{cc}
\underline{K}_{d, C B}^{q q} & 0 \\
0 & K_{d, C B}^{Y Y}
\end{array}\right]
$$

and

$$
\underline{\underline{M}}_{d, C B}=\left[\begin{array}{ll}
\underline{\underline{M}}_{d, C B}^{q q} & \underline{\underline{M}}_{d, C B}^{q Y} \\
\underline{\underline{M}}_{d, C B}^{Y q} & \underline{\underline{M_{d, C B}^{Y Y}}}
\end{array}\right] .
$$

To complete the blade-interface-disk modeling, it remains to relate the response of the interface degrees-of-freedom on the disk side to those on the blades. In this regard, two situations can be envisioned. In the first, the interface degrees-of-freedom of the disk are not collocated with those of the blades, see Fig. 1a, and a physical interface zone has been created. Assuming that there are no external forces acting on the interface zone of blade $b$, the disk-side and blade-side interfaces, $\underline{F}_{d, b}$ and $\underline{F}_{b}$ can be expressed as

$$
\begin{aligned}
& {\left[\begin{array}{c}
\underline{F}_{d, b} \\
\underline{F}_{b}
\end{array}\right]=\left[\begin{array}{ll}
\underline{\underline{M}} d b, d b & \underline{\underline{M}} d b, b \\
\underline{\underline{M}} b, d b & \underline{\underline{M}} b, b
\end{array}\right]\left[\begin{array}{c}
\underline{\underline{Y}}_{d, b} \\
\underline{\underline{Y}}_{b}
\end{array}\right]+\left[\begin{array}{ll}
\underline{K}_{d b, d b} & \underline{K}_{d b, b} \\
\underline{K}_{b, d b} & \underline{\underline{K}} b, b
\end{array}\right]\left[\begin{array}{c}
\underline{Y}_{d, b} \\
\underline{Y}_{b}
\end{array}\right]} \\
& =\underline{\underline{M}} d, b\left[\begin{array}{c}
\underline{\ddot{Y}}_{d, b} \\
\underline{\ddot{Y}}_{b}
\end{array}\right]+\underline{K}_{d, b}\left[\begin{array}{c}
\underline{Y}_{d, b} \\
\underline{Y}_{b}
\end{array}\right]
\end{aligned}
$$

where $\underline{\underline{M}}_{d b, d b}, \underline{\underline{M}} d b, b, \underline{\underline{M}}_{b, d b}$, etc. are the mass and stiffness matrices of the finite element model of the contact zone. Note that $\underline{M}_{b, b}$ and $\underline{K}_{b, b}$ are in general not equal to $\underline{M}_{b \text {,phys }}^{I I}$ and $\underline{K}_{b \text {, phys }}^{I I}$, although they relate to the same degrees-of-freedom, because they correspond to different finite element models, of the interface zone for the former matrices and of the blade for the latter ones.

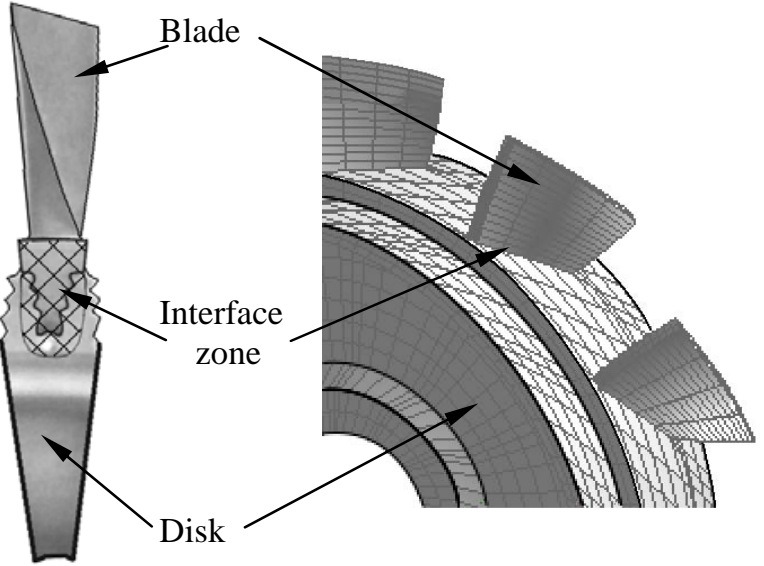

(a) (b)
Figure 1. Blade-Disk Interface Zone 
If a detailed finite element model of the interface zone has not been carried out, e.g. see Fig. 1b, it is still possible to introduce relative motions at the blade-disk interface by adopting Eq. (12) but with chosen mass and stiffness matrices $\underline{M}_{d b, d b}, \underline{\underline{M}} d b, b, \underline{\underline{M}}_{b, d b}$, etc. For example, assuming that there is only flexibility at the interface (no inertial effect), one can select

$$
\underline{\underline{M}}_{d b, d b}=\underline{\underline{M}}_{b, b}=\underline{\underline{M}} d b, b=\underline{\underline{M}} b, d b=\underline{=}
$$

and

$$
\underline{K}_{d b, d b}=\underline{K}_{b, b}=-\underline{\underline{K}} d b, b=-\underline{\underline{K}} b, d b=\underline{\underline{\hat{K}}}
$$

Without further information, it is convenient to select the matrix $\underline{\underline{K}}$ in terms of the stiffness matrices of the interface degrees-of-freedom on the blade ( $\left.\underline{K}_{b, p h y s}^{I I}\right)$ and disk ( $\left.\underline{K}_{d b, p h y s}^{I I}\right)$ sides as

$$
\hat{\underline{K}}=k\left(\underline{\underline{K}}_{b, \text { phys }}^{I I}+\underline{K}_{d b, \text { phys }}^{I I}\right)
$$

where the scalar $k$ is an adjustable, dimensionless parameter that describes the stiffness of the blade-disk interface.

The assembly of the various models, i.e. Eq. (8)-(12), provides the complete representation of the blade-interface-disk system. For example, for a disk supporting two blades, one would obtain the following mass and stiffness matrices associated with the degrees-of-freedom $\left(\underline{q}_{d}, \underline{Y}_{d, 1}, \underline{Y}_{1}, \underline{q}_{1}, \underline{Y}_{d, 2}, \underline{Y}_{2}, \underline{q}_{2}\right)$

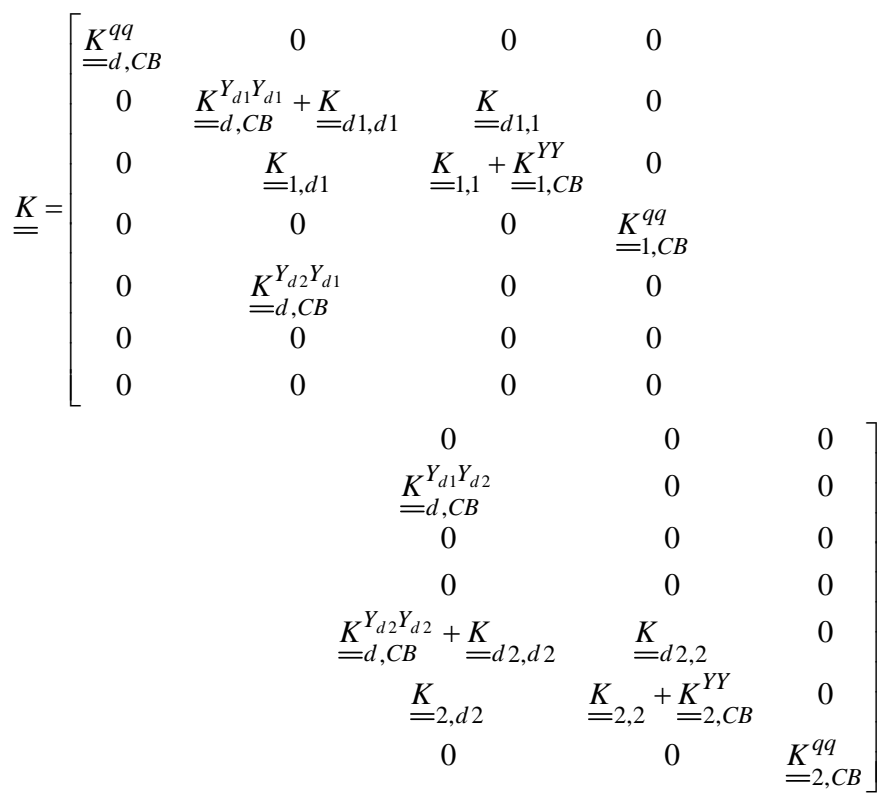

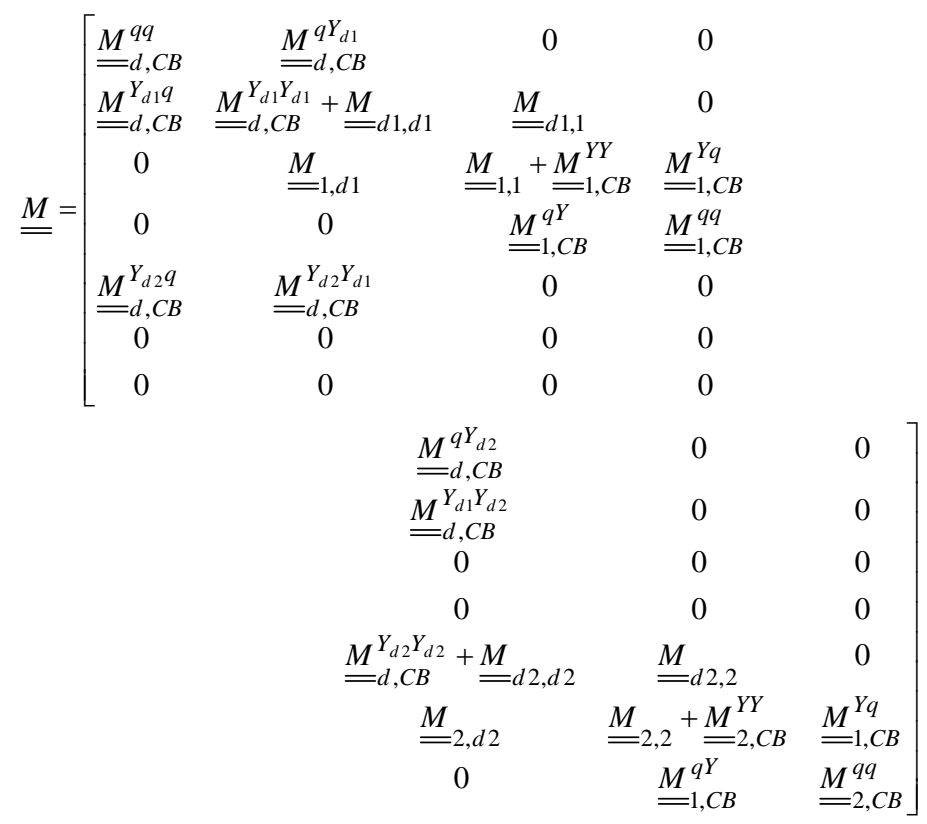

assuming that there is no direct coupling between two blades (e.g., no shroud).

\section{SIMULATION OF RANDOM INTERFACE PROPERTIES}

The discussion of the previous section has established a mean model, i.e. one in which there is no variation nor uncertainty from blade to blade. Such variations and uncertainties will be simulated next by randomizing the interface characteristic mass (if not zero, see Eq. (13)) and stiffness matrices $\underline{\underline{M}} d, 1, \underline{\underline{M}} d, 2, \ldots$ and $\underline{K}_{d, 1}, \underline{\underline{K}}{ }_{d, 2}, \ldots$ In this regard, note that in physical bladed disks, one would expect these matrices to vary both in magnitude (e.g. eigenvalues) and in form (e.g. eigenvectors) and thus it would be desirable to use a simulation strategy that performs a similar, complete randomization. The nonparametric stochastic modeling method (e.g., see [20-22]) is such an approach and accordingly will be selected here. It is briefly reviewed below for completeness.

Simulating random matrices $\underline{\underline{\underline{M}}} d, i$ and/or $\underline{\tilde{K}}_{d, i}$ requires effectively the specification of the joint probability density functions of their elements which represents an extraordinary amount of information that is generally not available. This difficulty has often in the past been resolved by further restricting the stochastic model of these matrices to affect only certain properties (e.g. the eigenvalues/natural frequencies but not the eigenvectors/modes) or by imposing a series of independence conditions between the various elements. All of these assumptions are ad hoc, without a firm physical basis, and thus may or may not lead to a correct perspective on the effects of variations/uncertainties.

In the nonparametric modeling approach, the lack of knowledge of the joint probability density function is resolved by selecting this function to achieve a maximum of the entropy under the physical constraints that the random matrix must satisfy, e.g. symmetry, positive definiteness. Through maximizing the entropy, the nonparametric modeling approach guarantees that the "tails" of the distribution will be as populated as possible, or equivalently that the simulated matrices will not simply represent small deviations around their mean values. Further, it has been shown that the simulated matrices are "fully" random, i.e. all 
of their elements, eigenvalues, and eigenvectors are random, as desired here.

It is necessary here that the simulated matrices $\underline{\underline{M}}_{d, i}$ and $\underline{\underline{K}}_{d, i}$ be symmetric, strictly positive definite (to avoid rigid body modes or zero mass modes), and further that their mean be given as the matrices $\underline{\underline{M}} d, i$ and/or $\underline{K}_{d, i}$ of Eq. (12) or Eq. (14). The problem of simulating matrices A satisfying these constraints and with a joint probability density function maximizing the entropy has been solved in [20] and exhibits an elegant solution. Specifically, denote by $\stackrel{\bar{A}}{=}$ the prescribed mean of $\stackrel{A}{=}$ and proceed first with its Cholesky factorization

$$
\underline{\bar{A}}=\underline{\underline{L}} \bar{L}^{T} \text {. }
$$

Then, the desired random matrices $\underset{=}{A}$ are obtained as

$$
\stackrel{A}{=}=\underline{\underline{L}} \underline{\underline{H}} \underline{H}^{T} \stackrel{{ }^{T}}{=}
$$

where $\underline{\underline{H}}$ denotes a lower triangular random matrix the elements of which are all statistically independent of each other, see Fig. 2. Further, the off-diagonal elements of $\underline{H}$ are zero mean Gaussian random variables with common standard deviation $\sigma=1 / \sqrt{\mu}$. Finally, the diagonal elements $H_{i i}$ are obtained as

$$
H_{i i}=\sqrt{\frac{Y_{i i}}{\mu}}
$$

where $Y_{i i}$ are Gamma random variables of parameter $p(i)=n-i+2 \lambda-1$. In these results, $n$ denotes the size the matrix $\stackrel{A}{=}$, $\mu=\frac{n+2 \lambda-1}{2}$, and $\lambda>0$ represents the single free parameter of the stochastic model. This parameter can be evaluated to meet any given information about the variability, e.g. standard deviation of appropriate natural frequencies, see the example section for discussion.

zero mean Gaussian, independent of each other with standard dev. $\sigma=1 / \sqrt{2 \mu}$

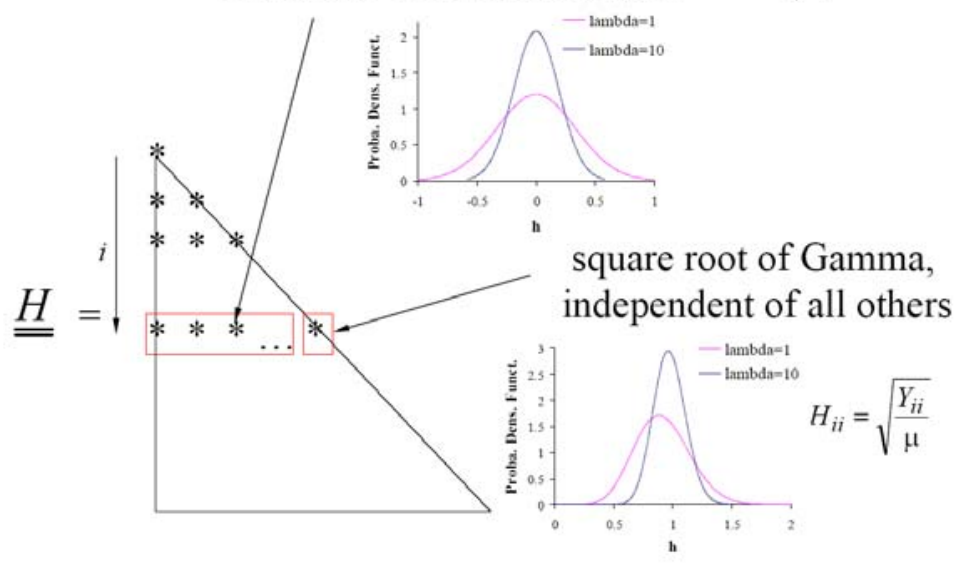

Figure 2: Structure of the random $\underline{\underline{H}}$ matrices (figures for $n=8, i=2$, and $\lambda=1$ and 10 ).

\section{EXAMPLE OF APPLICATION}

The above discussion was exemplified on the bladed disk model shown in Fig. 3 which is a modification (a reduction of the number of blades to 12) of the one considered in [5]. Since the original model is a blisk and thus does not exhibit a detailed modeling of the blade root as it fits in the disk, the simple interface modeling of Eq. (13) and (14) was adopted. The blade-interface-disk modeling was extracted directly from the sector finite element model using the cyclosymmetric CraigBampton formulation of [5].

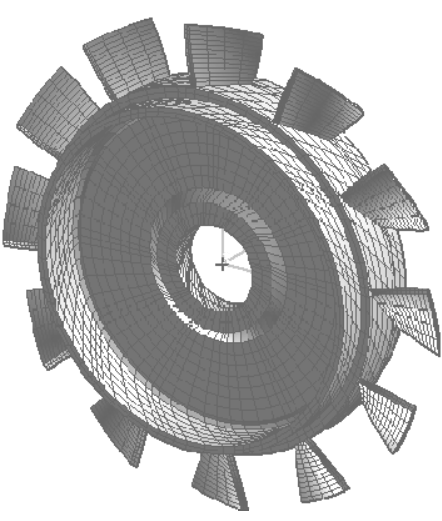

(a)

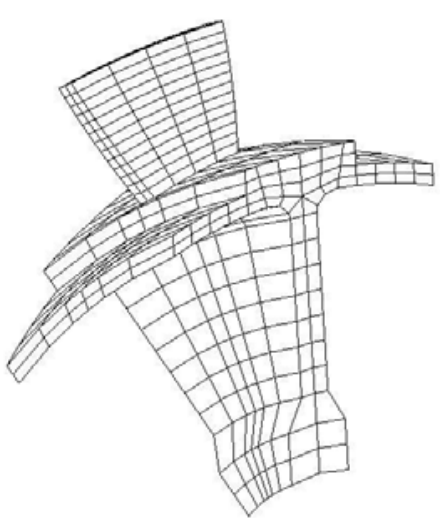

(b)

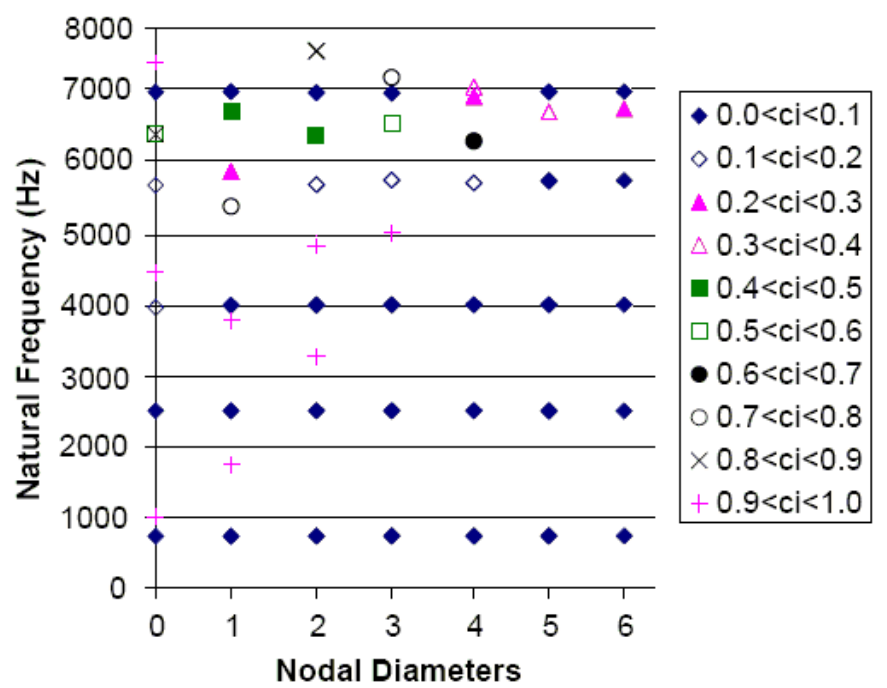

(c)

Figure 3. Bladed Disk example: (a) view, (b) blade sector finite element mesh, and (c) natural frequencies and coupling indices vs. nodal diameter plot.

The frequency vs. nodal diameter plot of Fig. 3(c) above has been enriched with a measure of blade to disk coupling recently introduced [22] and referred to as the coupling index (abbreviated by ci). This measure of coupling relates to the change in the cyclic system natural frequency corresponding to a particular number of nodal diameters induced by a change of blade only (not disk) Young's modulus. If the resonance condition investigated is associated with a blade alone motion, then a relative change $\delta E$ of blade Young's modulus would affect the blade alone natural frequencies by the factor $\sqrt{1+\delta E}$. In the opposite limit, if the motions induce deformations only in the disk, then 
the natural frequency will not be affected by $\delta E$. In general, the variations of the bladed disk natural frequencies will be between these two extreme cases and the coupling index will be defined as

$$
c i=\frac{\omega_{r}(1+\delta E)-\omega_{r}(1)}{\left(\sqrt{1+\delta E-1) \omega_{r}(1)}\right.}
$$

where $\omega_{r}(1+\delta E)$ corresponds to the appropriate bladed disk natural frequency associated with $r$ nodal diameter and a blade Young's modulus of $\mathrm{E}+\delta \mathrm{E}$. Similarly, $\omega_{r}(1)$ corresponds to $\delta E=0$.

The range of excitation frequencies extended over the range of the first blade alone natural frequencies which led to the selection of 20 blade alone modes in the reduced order model. An equal number of disk modes per sector were selected. Finally, all 45 degrees-of-freedom of the interface were retained. This reduced order model led to an excellent match of all frequencies below $7000 \mathrm{~Hz}$ as shown in Fig. 4. The damping ratio was set to $0.3 \%$ on all modes and the excitation was assumed to be a uniformly distributed loading on the blades only in the direction perpendicular to the disk.

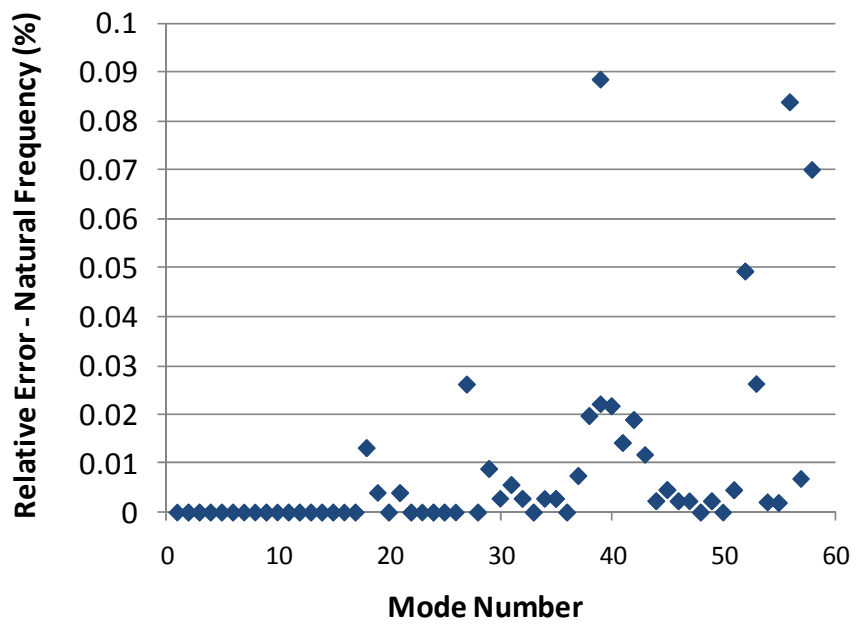

Figure 4. Relative error (\%) of the natural frequencies of the reduced order model as compared to cyclosymmetric finite element

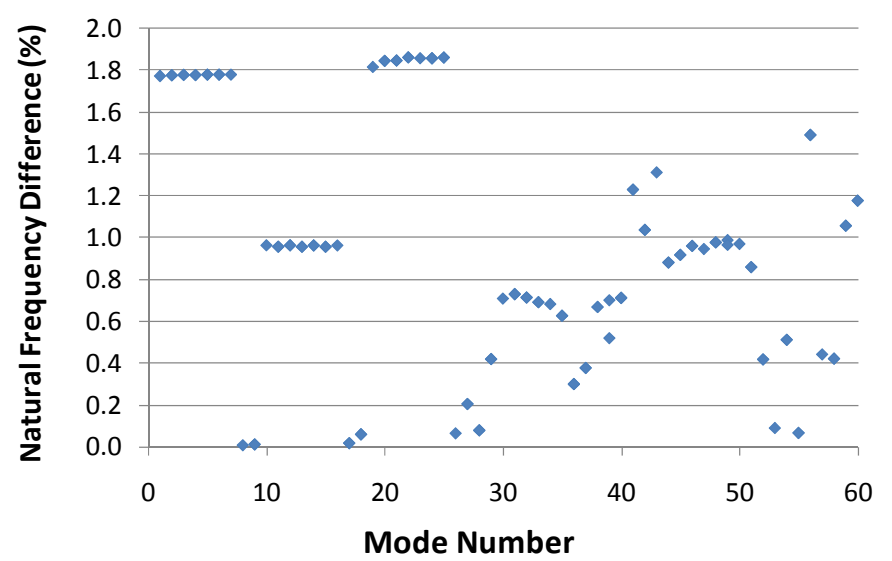

Figure 5. Relative difference (\%) of the natural frequencies of the reduced order model for $k=0.6$ vs. the $k=\infty$ values.

The selection of the parameter $k$ in Eq. (15) was considered next and the evolution of the tuned bladed disk natural frequencies was studied as $k$ was reduced from the limiting case $k=\infty$ corresponding to a perfectly stiff interface. It was found that for $k=0.6$ the tuned natural frequencies would typically be $1 \%$ lower than their counterparts for $k=\infty$, see Fig. 5 . This situation was considered to be representative of physical bladed disks and thus this value of $k$ was adopted for all ensuing computations.

The following computations focused on determining the amplification factor of the forced response of bladed disks with mistuned blade-disk interfaces. In addition to the nonparametric modeling approach described above, a simple strategy was also employed in which the parameter $k$ is varied from blade-to-blade as $\bar{k} V$ where $V$ is a random variable with probability density function

$$
p_{V}(v)=\frac{1}{4 \sigma \sqrt{3}} \frac{1}{\sqrt{v}} \text { for } v \in\left[(1-\sigma \sqrt{3})^{2},(1+\sigma \sqrt{3})^{2}\right] \text {. }
$$

Such a random variable is easily simulated from a uniform random variable $U$ in $[0,1]$ according to

$$
V=\left[1-2 \sigma \sqrt{3}\left(\frac{1}{2}-U\right)\right]^{2}
$$

Note that the term in bracket in Eq. (23) is a uniform random variable of mean equal to 1 and standard deviation $\sigma$. Then, $V$ is the square of a uniform random variable, and thus will be denoted as $U 2$ distributed in the remainder of this paper.

For the sake of comparisons, computations were also carried in which the interfaces were maintained tuned but the blade frequencies were varied. In these computations, both nonparametric method and $U 2$ random variations in natural frequencies were used.

Comparing computational results obtained under different modeling assumptions, i.e. random interfaces and random blade properties, requires the selection of a single physical parameter deemed most important in both conditions. In mistuning analyses, it is well recognized that the standard deviation of the blade alone natural frequencies represent this key parameter. Thus, the first set of comparisons were performed by plotting a particular statistics of the amplification factor vs. the standard deviation of the blade alone natural frequency for all 4 set of computations (interface nonparametric, interface $U 2$, blade nonparametric, and blade $U 2$ ).

Two more details must clarified: what statistic of the amplification factor should be used and for which blade alone natural frequency should the standard deviation be computed?

In regards to the choice of statistics for the amplification factor, it should be noted that the potentially dangerous situations are those in which a large amplification factor is achieved with a probability high enough to occur in a small population. Accordingly, it was proposed here to select as critical statistics the 95th percentile of the amplification factors, i.e. the amplification factor the exceedance of which occurs only with a probability of 0.05 .

The last question to address is of which natural frequency should the standard deviation be computed? In the frequency range of interest, i.e. [30,000-50,000] rad/sec or [4774-7958] Hz, the maximum amplitude of response of the tuned disk is achieved at a resonance associated with the 5th blade alone mode (or 5th family) for engine orders $0,1,2,5$, and 6 but with 4th blade alone mode (or 4th family) for engine orders 3 and 4 . Further, it was observed for the latter two engine orders that the resonance condition yielding the largest amplification factor in the presence of randomness in either blade alone properties or interface switched from the 4th family to the 5th one as the level of randomness increased.

For engine orders $0,1,2,5$, and 6 , it appears clearly that the 95th percentile of the amplification factor should be plotted vs. the standard 
deviation of the 5th blade alone natural frequency. For engine orders 3 and 4 , the situation appears less obvious. In this context, note that the switch of critical resonance condition appears for all 4 sets of computations in the same range of values of the standard deviation of the 5th natural frequency. Further, the standard deviation of the 4th blade alone natural frequency was found to be a one-to-one function of the corresponding statistic of the 5th blade alone natural frequency. These findings demonstrate that it is also appropriate to plot the 95th percentile of the amplification factor in engine orders 3 and 4 vs. the standard deviation of the 5th blade alone natural frequency. They also show however that the plots obtained with engine orders 3 and 4 can be compared to each other but not to those corresponding to engine orders 0 , $1,2,5$, and 6 which nevertheless can be compared to each other.

With these clarifications, shown in Figs 6-12 are the 95th percentiles of the amplification factor corresponding to engine orders 0-6 in the frequency range [4774-7958] $\mathrm{Hz}$ vs. the standard deviation of the 5th blade alone natural frequency.

From these results, it appears that the mistuning of the blade-disk interface leads rather consistently to a larger amplification factor than does the traditional blade alone frequencies mistuning.

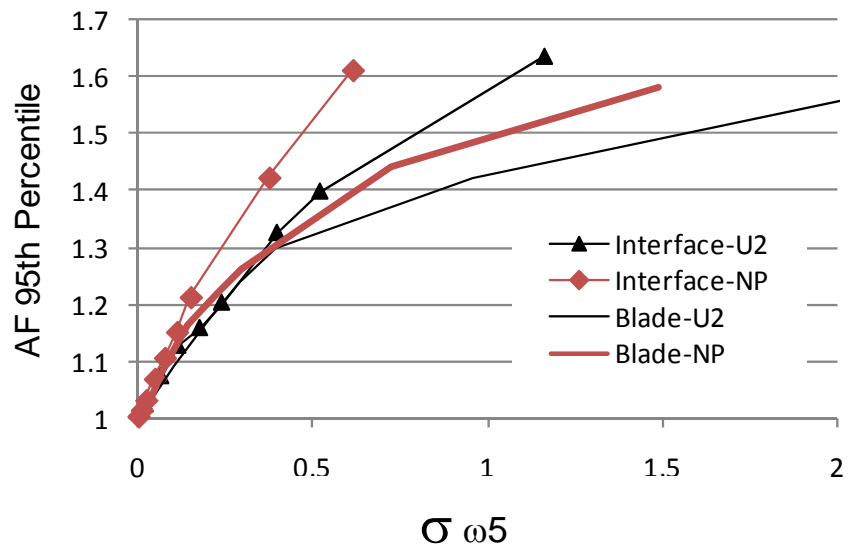

Figure 6. 95th percentile of the amplification factor vs. standard deviation of the 5 th blade alone natural frequency (in $\%)$, engine order 0 excitation in the range [4774-7958] $\mathrm{Hz}$.

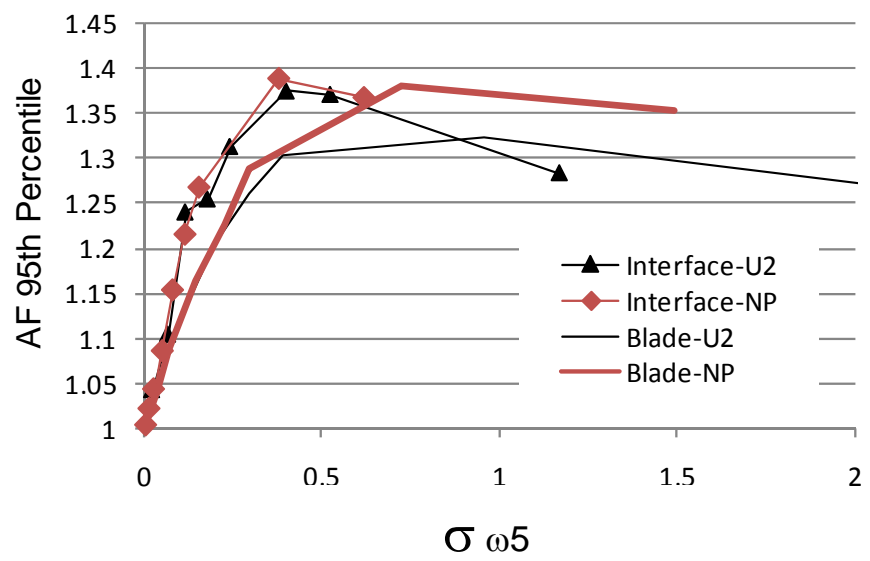

Figure 7. 95th percentile of the amplification factor vs. standard deviation of the 5 th blade alone natural frequency (in $\%)$, engine order 1 excitation in the range [4774-7958] $\mathrm{Hz}$.

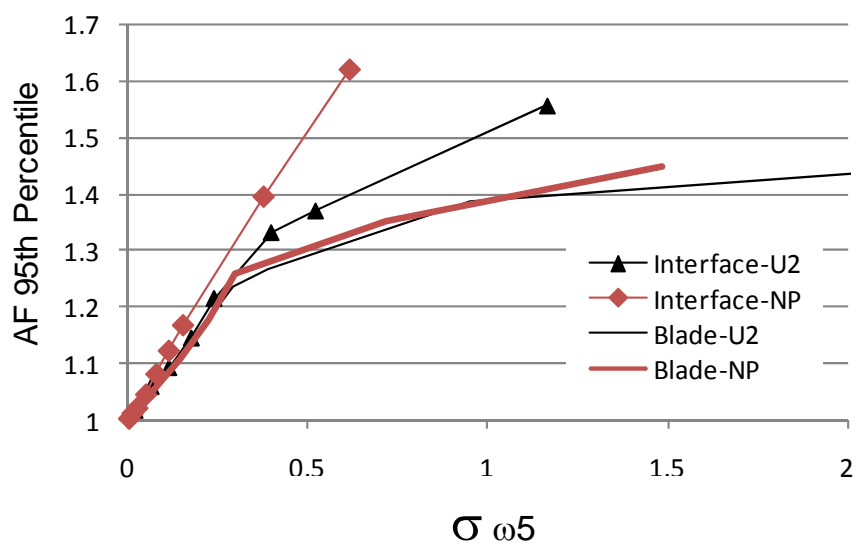

Figure 8. 95th percentile of the amplification factor vs. standard deviation of the 5th blade alone natural frequency (in \%), engine order 2 excitation in the range [4774-7958] $\mathrm{Hz}$.

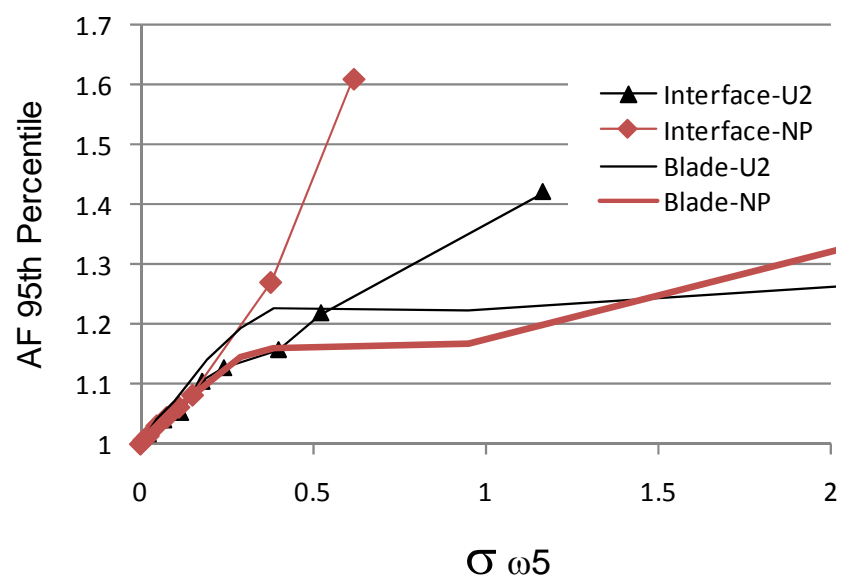

Figure 9. 95th percentile of the amplification factor vs. standard deviation of the 5 th blade alone natural frequency (in \%), engine order 3 excitation in the range [4774-7958] Hz.

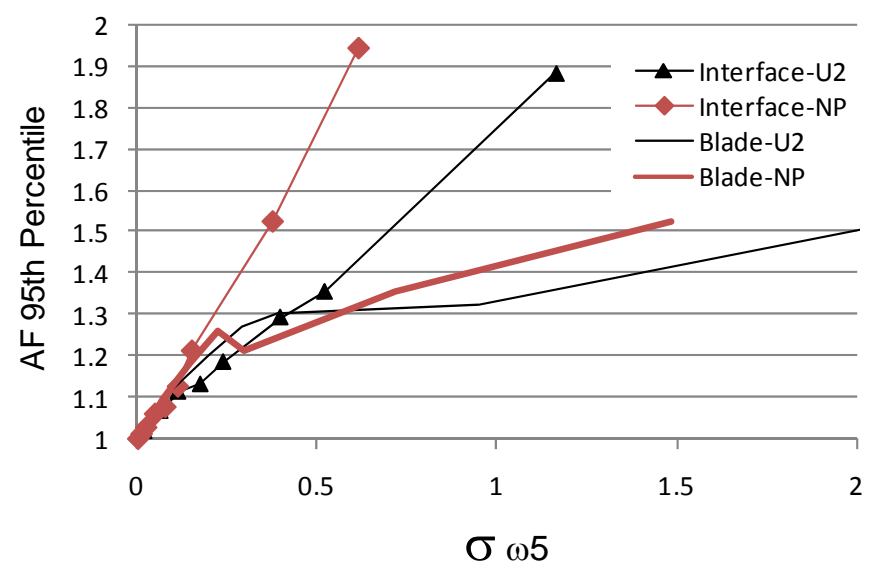

Figure 10. 95th percentile of the amplification factor vs. standard deviation of the 5th blade alone natural frequency (in \%), engine order 4 excitation in the range [4774-7958] Hz. 


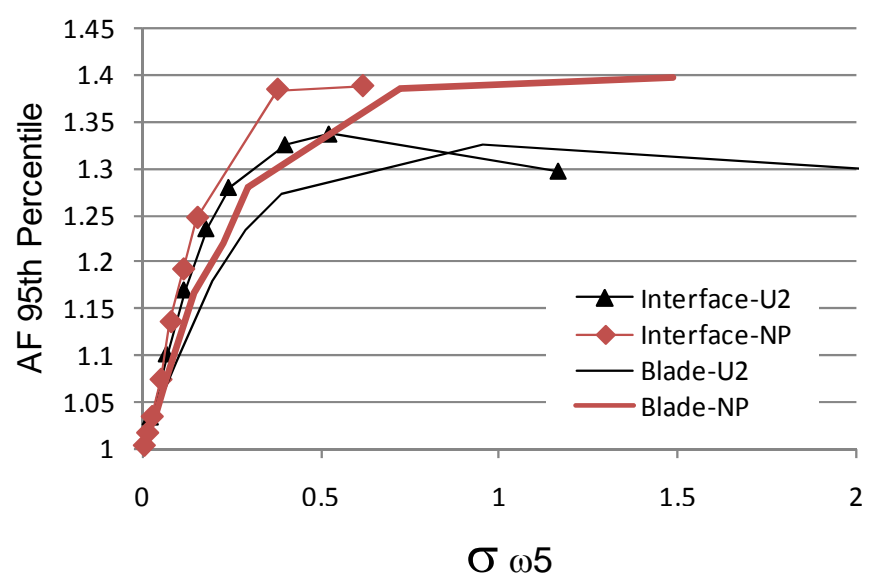

Figure 11. 95th percentile of the amplification factor vs. standard deviation of the 5 th blade alone natural frequency (in $\%)$, engine order 5 excitation in the range [4774-7958] Hz.

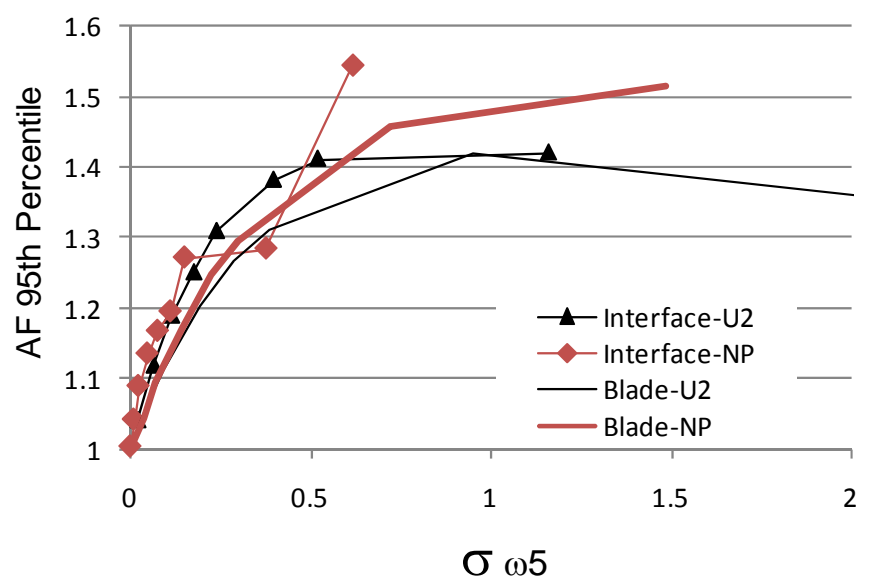

Figure 12. 95th percentile of the amplification factor vs. standard deviation of the 5 th blade alone natural frequency (in $\%)$, engine order 6 excitation in the range [4774-7958] $\mathrm{Hz}$.

In seeking an explanation for this unexpected behavior, it was first observed that the distribution of the 5th blade alone natural frequencies obtained by interface and blade alone mistuning were different even though they exhibited the same standard deviation. Specifically, it was found that the distributions of these frequencies obtained from random interfaces exhibited, at the contrary of the blade mistuning cases, a shift of the mean toward smaller frequencies. This mean shift was found to be approximately $0.7 \%$ at the highest level of blade alone frequency standard deviation. Since the difference between the 95th percentiles of the amplification factor obtained with random interfaces and with random blade properties are noticeably larger than $0.7 \%$ in those situations, it would appear that this mean shift is not the cause of the higher amplification factors.

The reason for the mean shift is in fact quite clear: increasing the stiffness of the interface (e.g. by increasing $k$ ) will typically only produce a small increase in frequency as this parameter is limited on the high side by its rigid interface, $k=\infty$, limit which is just $1 \%$ above. On the contrary, there is no constraining limit on the softening side and much larger decrease in blade alone natural frequencies can be produced. This discussion also clarifies the limited range of standard deviations of blade alone natural frequencies that can be produced with the random interface model.

To provide further evidence to rule out the mean shift as the cause for the higher amplification factor, it was decided to simulate random interface properties to produce a mean natural frequency either constant or increasing with the corresponding standard deviation. This step was achieved simply by proceeding with a nonparametric modeling of $\underline{\underline{K}}_{d, i}^{-1}$ instead of $\underline{\underline{K}}_{d, i}$ as done for Figs. 6-12. With this revised approach, the mean value does increase slightly with increasing standard deviation. The logic for this behavior is that the distribution of the eigenvalues of the random matrix simulated by the nonparametric approach shifts to the left for small values of $\lambda$ leading to larger eigenvalues of the inverse. The use of $\underline{\underline{K}}_{d, i}^{-1}$ thus favor the stiffening of the interface and increasing mean natural frequencies are observed. The disadvantage of the $\underline{\underline{K}}_{d, i}^{-1}$ simulation strategy is that the range of standard deviation of blade alone natural frequencies that can be produced is even further reduced as compared to the scheme using $\underline{\underline{K}}_{d, i}$. Forced response computations carried out with the $\underline{\underline{K}}_{d, i}^{-1}$ based simulation algorithm at the highest possible standard deviation of the 5th blade alone natural frequency led to a 95th percentile of response smaller than obtained for the $\underline{\underline{K}}_{d, i}$ based scheme for 5 of the 7 engine orders $0-6$. For two of these engine orders, a higher 95th percentile of the amplification factor was obtained with a mean blade alone natural frequency higher than for the mean model.

Based on this result and the small magnitude of the mean shift obtained $(0.7 \%)$ it is expected that this shift does not contribute significantly to the higher amplification factors observed in Figs. 6-12.

Since the blade alone natural frequency does not appear to provide an explanation for this increased response, it is quite natural to question whether a variability in the blade alone mode shapes could be the underlying factor. To this end, shown in Figs 13 and 14 are the standard deviation of the mode shape norm $\left\|\underline{\psi}_{i}-\underline{\Psi}_{i}\right\|$ where $\underline{\psi}_{i}$ and $\underline{\Psi}_{i}$ are the $i$ th mass normalized mode shapes of the random and mean blade, respectively, obtained for all 4 modeling strategies. Clearly, the standard deviations obtained with random interfaces are much larger than those obtained by varying simply the blade properties thus supporting the tentative role of the mode shape variability to explain the increased amplification factors in Figs. 6-12. In analyzing the results of Figs 13 and 14 , it should be noticed that the nonparametric method consistently lead to a higher standard deviation of mode shape norm than the use of a single random variable. This finding applies to both random interface as well as random blade properties.

The above discussion indicates that both blade alone natural frequency and mode shape variations affect the amplification factor and thus a different key parameter should be used in comparing the random interface results with those obtained with random blade alone properties. In this regard, note that the mode shape variability will play at the assembly of the different sectors to form the blade disk which suggests that the key parameter sought may be a disk property, not a blade alone feature. This key parameter must however exhibit a fairly simple physics if it is to be a useful indicator and this required simplicity rules out the use of natural frequencies and/or mode shapes of the fully mistuned disks. 


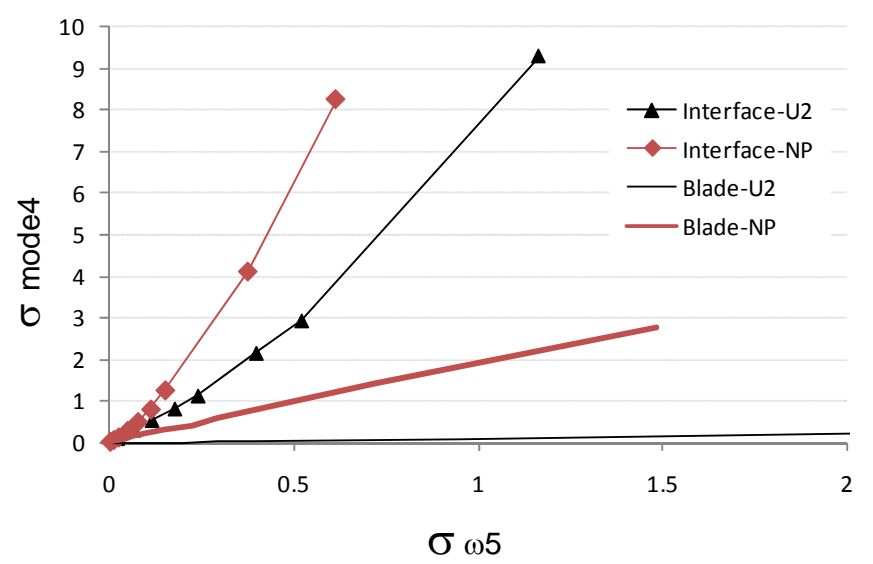

Figure 13. Standard deviation of blade alone mode shape deviation vs. standard deviation of the 5 th blade alone natural frequency (in \%), mode 4.

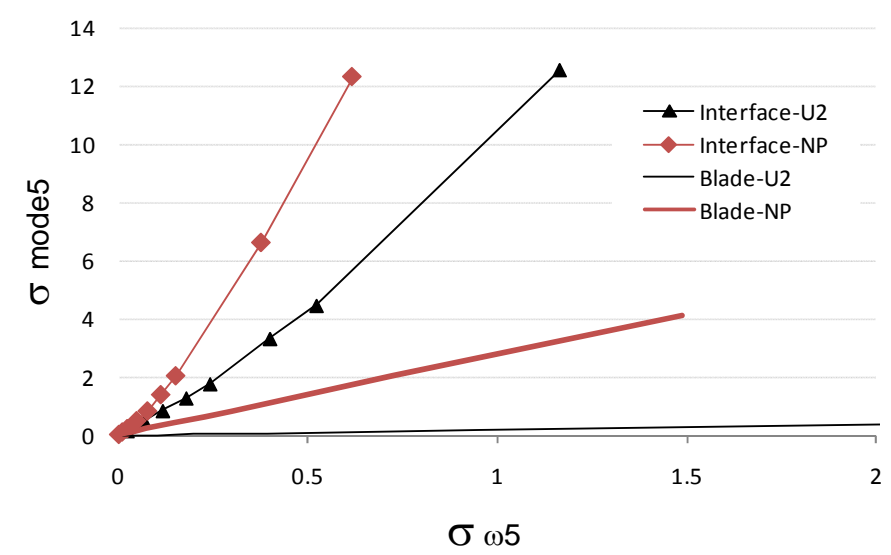

Figure 14. Standard deviation of blade alone mode shape deviation vs. standard deviation of the 5 th blade alone natural frequency (in \%), mode 5.

Of complexity intermediate to that of mistuned blades alone and fully mistuned bladed disks are uniformly mistuned disks which are cyclic structures built with identical blades but different from those of the mean model. Uniformly mistuned bladed disks exhibit, as their tuned counterparts, natural frequencies associated with nodal diameters and thus provide a tentative basis to assess nodal diameter effects of mistuning. Accordingly, shown in Figs 15-21 are the 95th percentiles of the amplification factor induced by $r$ th engine order excitations for $r=0-6$ plotted vs. the standard deviation of the corresponding $r$ th nodal diameter natural frequency of uniformly mistuned disks. The 4 curves shown on each plot correspond to the nonparametric and $U 2$ random interfaces and nonparametric and $U 2$ blade alone properties. Note the differences between the 4 curves are typically reduced as compared to those shown in Figs 6-12 suggesting that the standard deviation of the natural frequencies of uniformly mistuned disks is in fact a better parameter to assess the effects of various types of mistuning than the corresponding statistics of blades alone.

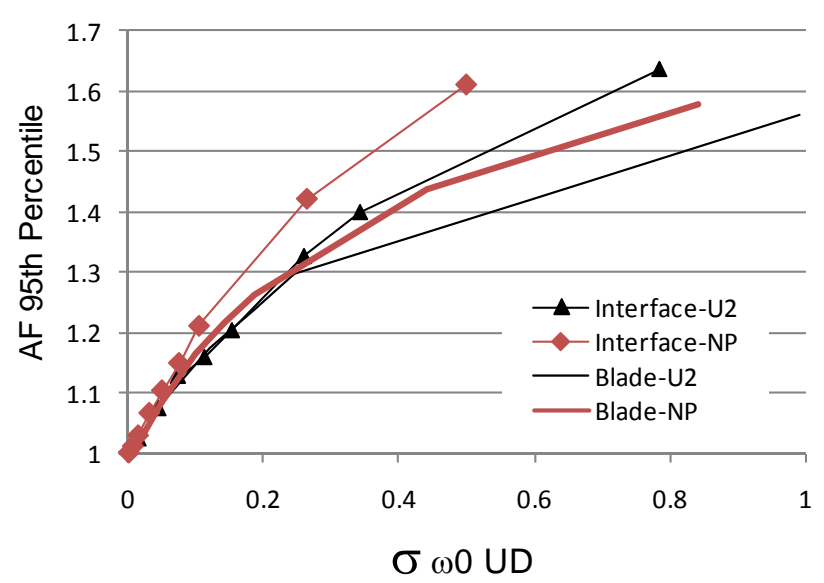

Figure 15. 95th percentile of the amplification factor vs. standard deviation of the 0th nodal diameter natural frequency of uniformly mistuned disk (in \%), engine order 0 excitation in the range [4774-7958] $\mathrm{Hz}$.

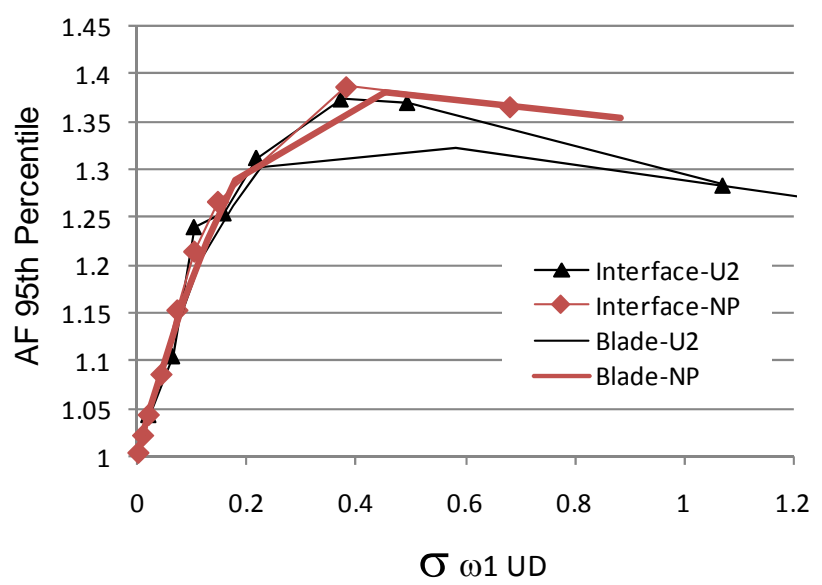

Figure 16. 95th percentile of the amplification factor vs. standard deviation of the 1st nodal diameter natural frequency of uniformly mistuned disk (in \%), engine order 1 excitation in the range [4774-7958] $\mathrm{Hz}$.

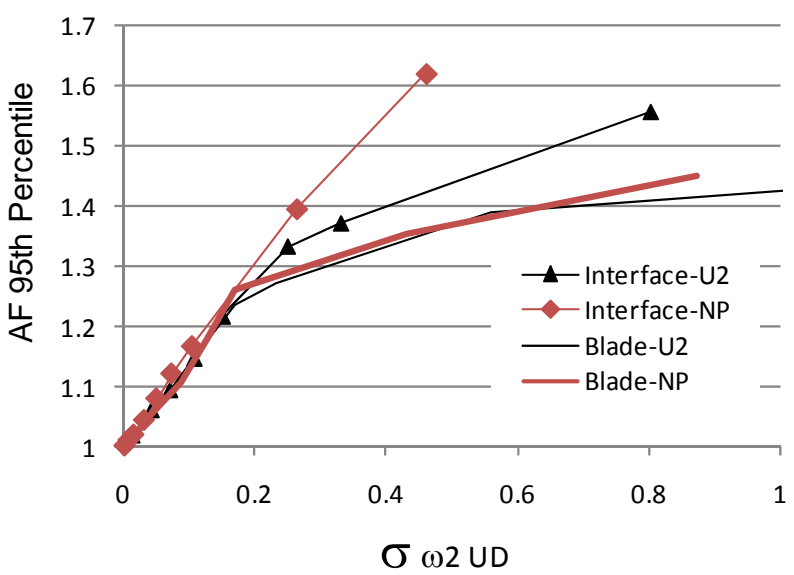

Figure 17. 95th percentile of the amplification factor vs. standard deviation of the 2 nd nodal diameter natural frequency of uniformly mistuned disk (in \%), engine order 2 excitation in the range [4774-7958] $\mathrm{Hz}$. 


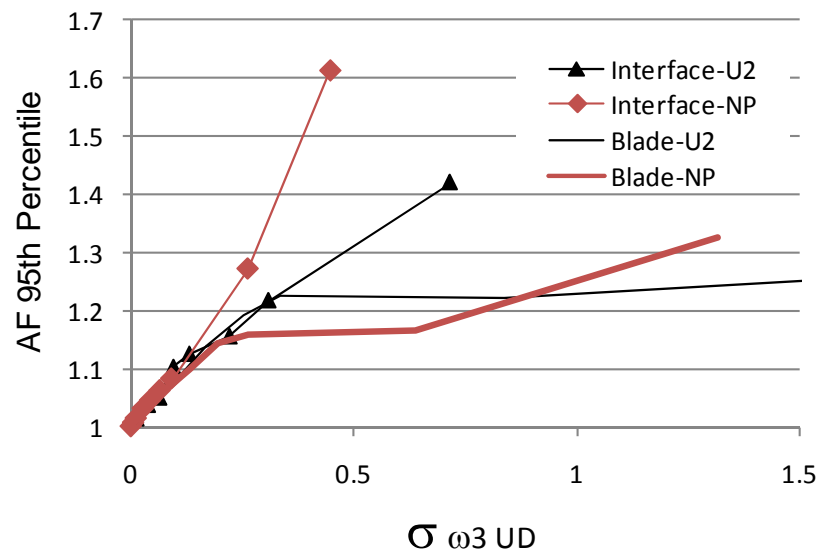

Figure 18. 95th percentile of the amplification factor vs. standard deviation of the 3rd nodal diameter natural frequency of uniformly mistuned disk (in \%), engine order 3 excitation in the range [4774-7958] $\mathrm{Hz}$.

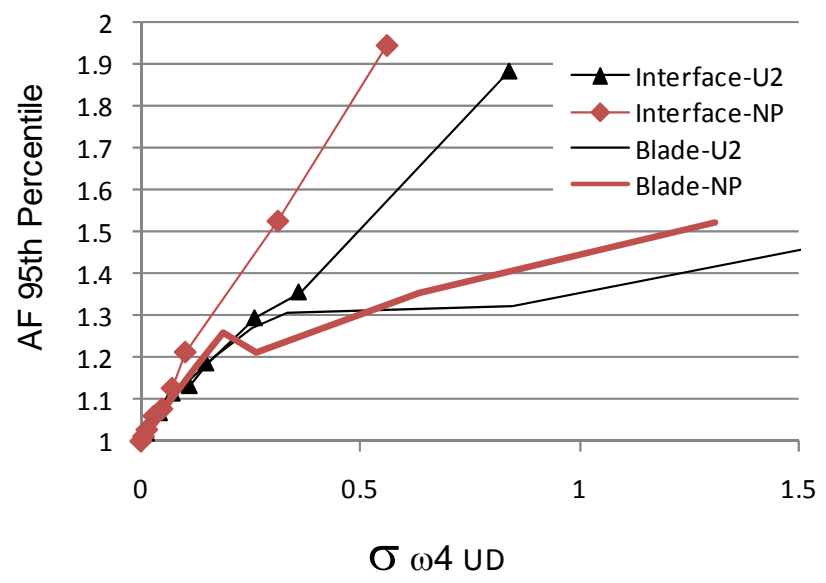

Figure 19. 95th percentile of the amplification factor vs. standard deviation of the 4th nodal diameter natural frequency of uniformly mistuned disk (in \%), engine order 4 excitation in the range [4774-7958] $\mathrm{Hz}$.

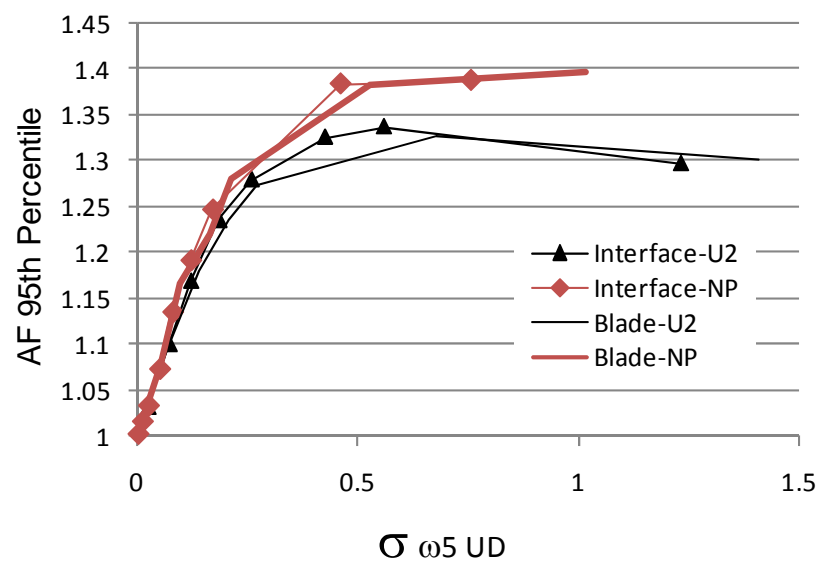

Figure 20. 95th percentile of the amplification factor vs. standard deviation of the 5 th nodal diameter natural frequency of uniformly mistuned disk (in \%), engine order 4 excitation in the range [4774-7958] $\mathrm{Hz}$.

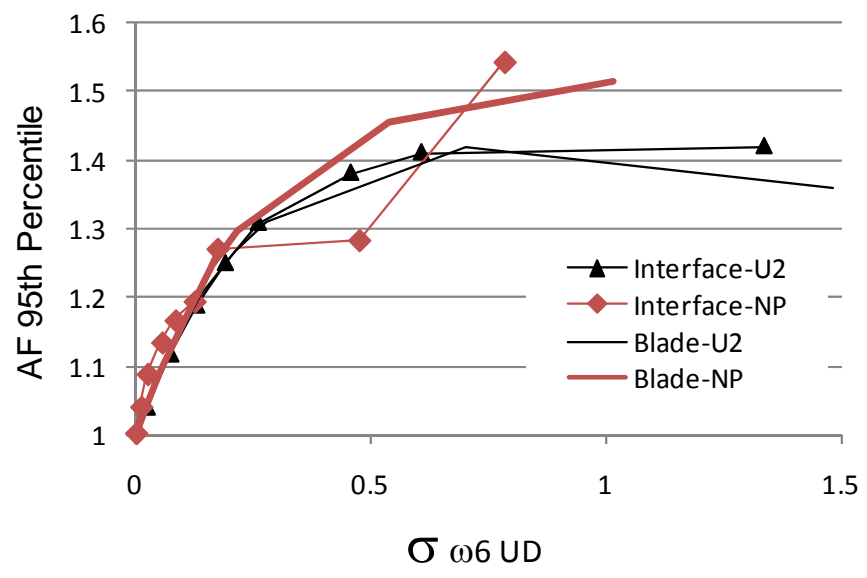

Figure 21. 95th percentile of the amplification factor vs. standard deviation of the 6 th nodal diameter natural frequency of uniformly mistuned disk (in \%), engine order 6 excitation in the range [4774-7958] $\mathrm{Hz}$.

\section{SUMMARY}

This paper focuses on the determination of the effects on the forced response of bladed disks of small variations (mistuning) in the bladedisk interface properties. A blade-interface-disk mean model was first developed that relies on both the Craig-Bampton approach and a local modeling of the interface, see Eq. (12)-(15). Then, both model and data uncertainties were introduced in this model using the nonparametric stochastic modeling approach. An example of application was next considered to exemplify these concepts and provide a comparison with the more classical blade mistuning effects. In this effort, it was demonstrated that

(i) the mistuning of the blade-disk interfaces leads, at equal variability on the blade alone frequencies, to larger amplification factors than obtained by simply mistuning the blade natural frequencies, and

(ii) that the nonparametric approach leads typically to larger amplification factors than obtained with a simple uniform distribution of properties on the blades, in regards to both interface and blade alone properties.

Both of these findings were explained by the increased variability of the blade alone mode shapes exhibited by the blades with random interfaces as compared to those with random blade alone properties.

Finally, it was suggested that the standard deviation of the natural frequencies of uniformly mistuned bladed disks are better predictors of the mistuned response than the corresponding statistics of blades alone.

These results demonstrate the importance of considering mistuning in blade-disk interface and the need to treat it separately from blade alone mistuning.

\section{ACKNOWLEDGMENTS}

The support of this work by a fellowship from CONACyT to the first author is gratefully acknowledged. In addition, the authors wish to express their appreciation to Professor C. Pierre and Drs M.P. Castanier and R. Bladh, for the use of the blisk example geometry.

\section{REFERENCES}

[1] Wei, S. T., and Pierre, C., 1988a, "Localization Phenomena in Mistuned Assemblies with Cyclic Symmetry - Part I: Free Vibrations,” 
Journal of Vibration, Acoustics, Stress, and Reliability in Design, Vol. 110, No. 4, pp. 429-438.

[2] Wei, S. T., and Pierre, C., 1988b, "Localization Phenomena in Mistuned Assemblies with Cyclic Symmetry - Part II: Forced Vibrations," Journal of Vibration, Acoustics, Stress, and Reliability in Design, Vol. 110, No. 4, pp. 439-449.

[3] Rivas-Guerra, A.J., and Mignolet, M.P., "Local/Global Effects of Mistuning on the Forced Response of Bladed Disks," Journal of Engineering for Gas Turbines and Power, Vol. 126, No. 1, pp. 131-141, 2004.

[4] Castanier, M.P., Ottarson, G., and Pierre, C., "A Reduced Order Modeling Technique for Mistuned Bladed Disks," Journal of Vibration and Acoustics, 119, pp. 439-447, 1997.

[5] Bladh, R., Castanier, M. P., and Pierre, C., "Component-Mode-Based Reduced Order Modeling Techniques for Mistuned Bladed Disks-Part I: Theoretical Models,” Journal of Engineering for Gas Turbines and Power, 123(1), pp. 89-99, 2001.

[6] Yang, M.-T., and Griffin, J. H., 2001, “A Reduced Order Model of Mistuning Using a Subset of Nominal System Modes,'” Journal of Engineering for Gas Turbines and Power, 123(4), pp. 893-900.

[7] Petrov, E. P., Sanliturk, K. Y., and Ewins, D. J., 2002, "A New Method for Dynamic Analysis of Mistuned Bladed Disks Based on Exact Relationship Between Tuned and Mistuned Systems," Journal of Engineering for Gas Turbines and Power, 122, pp. 586-597.

[8] Whitehead, D.S., 1966, "Effect of Mistuning on the Vibration of Turbomachine Blades Induced by Wakes,” Journal of Mechanical Engineering Science, 8, pp. 15-21.

[9] Whitehead, D.S., 1998, "The Maximum Factor by Which Forced Vibration of Blades Can Increase Due to Mistuning," Journal of Engineering for Gas Turbines and Power, 120, pp. 115-119.

[10] Whitehead, D.S., 1976, "Effect of Mistuning on Forced Vibration of Blades with Mechanical Coupling," Journal of Mechanical Engineering Science, 18, pp. 306-307.

[11] Rivas-Guerra, A.J., and Mignolet, M.P. 2003, "Maximum Amplification of Blade Response due to Mistuning: Localization and Mode Shapes Aspects of the Worst Disks,” Journal of Turbomachinery, 125(3), pp. 442-454.

[12] Kenyon, J.A., Griffin, J.H., Feiner, D.M., 2003, “Maximum Bladed Disk Forced Response from Distortion of a Structural Mode,” Journal of Turbomachinery, 125(2), pp. 352-363.

[13] Xiao, B., Rivas-Guerra, A.J., and Mignolet, M.P., 2004, “Maximum Amplification of Blade Response due to Mistuning in Multi-Degree-ofFreedom Blade Models,” Presented at the IGTI Turbo Expo’04, Vienna, Austria, Jun. 14-17, ASME Paper GT-2004-54030. To Appear Journal of Turbomachinery.

[14] Han, Y., Xiao, B., Mignolet, M.P., "Expedient Estimation of the Maximum Amplification Factor in Damped Mistuned Bladed Disks," Presented at the IGTI Turbo Expo'07, Montreal, Canada, May 14-17, 2007, ASME Paper GT-2007-27353. To Appear Journal of Turbomachinery

[15] C. Martel and R. Corral, "Asymptotic Description of Maximum Mistuning Amplification of Bladed-Disk Forced Response,” Journal of Engineering for Gas Turbines and Power, Vol. 131, 022506-1

[16] Segui, B., Casanova, E., 2007, "Application of a Reduced Order Modeling Technique for Mistuned Bladed Disk-Shaft Assemblies," Presented at the IGTI Turbo Expo'07, Montreal, Canada, May 14-17, 2007, ASME Paper GT2007-27594.

[17] Laxalde, D., Lombard, J.P., and Thouverez, F., “2007, Dynamics of Multi-Stage Bladed Disks Systems," Presented at the IGTI Turbo Expo'07, Montreal, Canada, May 14-17, 2007, ASME Paper GT200727083
[18] Sinha, A., 2007, "Reduced-Order Model of a Mistuned Multi-Stage Bladed Rotor,” Presented at the IGTI Turbo Expo'07, Montreal, Canada, May 14-17, 2007, ASME Paper GT2007-27277.

[19] Song, S. H., Castanier, M.P., Pierre, C., 2007, "System Identification Of Multistage Turbine Engine Rotors," Presented at the IGTI Turbo Expo'07, Montreal, Canada, May 14-17, 2007, ASME Paper GT2007-28307.

[20] Soize, C., 2000, “A Nonparametric Model of Random Uncertainties on Reduced Matrix Model in Structural Dynamics," Probabilistic Engineering Mechanics, Vol. 15, No. 3, pp. 277-294.

[21] Capiez-Lernout, E., and Soize C., "Nonparametric Modeling of random uncertainties for dynamic response of mistuned bladed disks," Journal of Engineering for Gas Turbines and Power, 126 (3), pp. 610618, 2004.

[22] Capiez-Lernout, E., Soize, C., Lombard, J.P., Dupont, C., and Seinturier, E., 2005, "Blade Manufacturing Tolerances Definition for a Mistuned Industrial Bladed Disk," Journal of Engineering for Gas Turbines and Power, Vol. 127, No. 3, pp. 621-629.

[23] Kiflu, B., "Analysis of Mistuned Bladed Disks by a Reduced Order Technique: Validation, Identification, and Stochastic Modeling,” M.S., Arizona State University, May 2005. 